\title{
Overview of the BINP accelerator complex
}

\author{
Dmitry Shwartz ${ }^{1,2 *}$, Fedor Emanov ${ }^{1,2}$ and Pavel Piminov ${ }^{1}$ \\ ${ }^{1}$ Budker Institute of Nuclear Physics, 630090 Novosibirsk, Russia \\ ${ }^{2}$ Novosibirsk State University, 630090 Novosibirsk, Russia
}

\begin{abstract}
The overview of two running BINP electron-positron colliders VEPP-4M and VEPP-2000 is presented. Since 2016 both colliders are joint to the single accelerator complex via common injector that supplies more intensive beams. In 2017 VEPP-2000 started data taking after 3 years of upgrade. The project of Super-Charm-Tau factory waits for the final government's approval being considered as one of 6 mega-science project in Russia.
\end{abstract}

\section{Introduction}

Budker Institute of Nuclear Physics (BINP) has a long history of experiments at colliders. Starting from pioneer electron-electron machine VEP-1 in early 1960s several electronpositron colliders served for several generations of particle detectors. Presently two operating colliders VEPP-4M and VEPP-2000 are joint via single new BINP Injection Complex (IC) and cover in common energy range from 0.16 to $5.5 \mathrm{GeV}$ per beam.

\section{Injection Complex}

Injection Complex VEPP-5 is a linear accelerator based $\mathrm{e}+\mathrm{e}-$ beams source with Damping Ring (DR). It consists of $270 \mathrm{MeV}$ driving electron Linac, $510 \mathrm{MeV}$ positron Linac and DR. DR stores and cools down both electron and positron beams for the next extraction to K-500 beam transfer line (see Fig 1). Key designed parameters of IC are presented in Table 1.

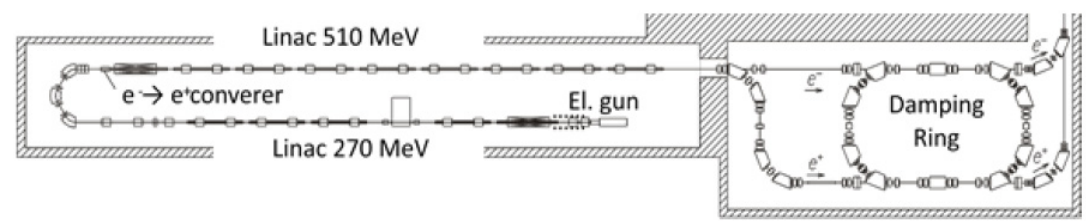

Fig. 1. VEPP-5 Injection Complex layout [1,2].

Table 1.VEPP-5 Injection Complex Design Parameters [1].

\begin{tabular}{|l|l|}
\hline Energy (2016/17 run) & $385-420 \mathrm{MeV}$ \\
\hline
\end{tabular}

\footnotetext{
* Corresponding author: d.b.shwartz@inp.nsk.su
} 


\begin{tabular}{|l|c|}
\hline Electron storage rate & $2 \times 10^{10} / \mathrm{s}$ \\
\hline Positron storage rate & $2 \times 10^{9} / \mathrm{s}$ \\
\hline Repetition rate & $12.5 \mathrm{~Hz}$ \\
\hline Maximum $\mathrm{e}^{-} / \mathrm{e}+$ extracted bunch intensity & up to $1.2^{*} 10^{11}$ \\
\hline
\end{tabular}

\section{VEPP-4 facility}

VEPP-4 is a multipurpose facility [3, 4] with several research programs including high energy physics (HEP) at colliding $\mathrm{e}^{+} \mathrm{e}^{-}$beams, nuclear physics, synchrotron radiation (SR), accelerator physics study, etc. VEPP-4 consists of the VEPP-3 and VEPP-4M storage rings and the pulsed-magnet beam transfer line from VEPP-3 to VEPP-4M. The VEPP-4 facility is shown in Fig. 2, main parameters are listed in Table 2.

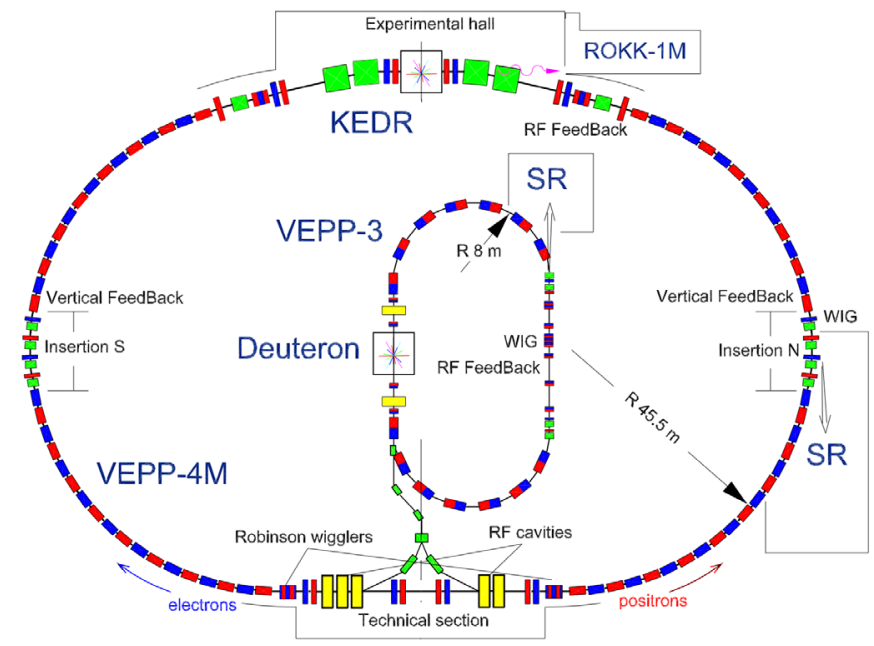

Fig. 2. VEPP-4 accelerator complex layout.

Table 2. Parameters of VEPP-3 at $2 \mathrm{GeV}$ and VEPP-4M at $1.8 \mathrm{GeV}$.

\begin{tabular}{|l|l|l|}
\hline Parameter & VEPP-3 & VEPP-4M \\
\hline Circumference & $74.4 \mathrm{~m}$ & $366 \mathrm{~m}$ \\
\hline Energy range & $0.4-2 \mathrm{GeV}$ & $1 \div 5.2 \mathrm{GeV}$ \\
\hline Betatron tunes & $5.12 / 5.18$ & $8.54 / 7.58$ \\
\hline Nat. chroms & $-3 /-2$ & $-14 /-20$ \\
\hline Hor. emittance & $250 \mathrm{~nm} \cdot \mathrm{rad}$ & $20 \mathrm{~nm} \cdot \mathrm{rad}$ \\
\hline Energy spread & $7 \cdot 10^{-4}$ & $4 \cdot 10^{-4}$ \\
\hline Bunch length & $9 \mathrm{~cm}$ & $6 \mathrm{~cm}$ \\
\hline
\end{tabular}

Since 2016 VEPP-4 uses IC connected to VEPP-3 with long beam transfer line. The injection rate is $1 \mathrm{~Hz}$. The positron production was increased tenfold in comparison with the old injector [5]. It allowed to increase the peak luminosity of VEPP-4M from $2 \cdot 10^{30} \mathrm{~cm}^{-2} \mathrm{~s}^{-1}$ to $3.3 \cdot 10^{30} \mathrm{~cm}^{-2} \mathrm{~s}^{-1}$ for $\mathrm{psi}(3770)[6]$. 
VEPP-3 operates either as the booster for VEPP-4M (the injection energy $\sim 1.8 \mathrm{GeV}$ ) or independently for SR and nuclear physics research (with the maximum energy up to $2 \mathrm{GeV}$ ). There are ten SR user stations at VEPP-3 that use radiation from the bending magnet and the normal conducting wavelength shifter.

VEPP-4M is an $\mathrm{e}^{+} \mathrm{e}^{-}$collider with the KEDR detector [7] operating in the wide beam energy range from $0.92 \mathrm{GeV}$ to $5.2 \mathrm{GeV}$. The low energy run $(0.9 \div 1.9 \mathrm{GeV})$ started in 2000 has been completed. The results include precision mass measurements of tau lepton, $\psi$ and D mesons, measurements of parameters of $\psi$ and $\eta_{\mathrm{c}}$ mesons, search for narrow resonances, measurements of the ratio of the hadronic cross section, etc. The next run will be in the high energy range of VEPP-4M (from 2 to $5 \mathrm{GeV}$ ) for R-scan, $\Upsilon$-meson study and $\gamma \gamma$-physics. For this purpose upgrade of the facility is carrying out (new PS, RF system upgrade, control system, etc.). SR experiments are performed at VEPP-4M in two modes: VUV and soft Xrays at the low energy of $1.9 \mathrm{GeV}$ from the bending magnet and hard X-rays at $4 \mathrm{GeV}$ with radiation from the $12 \mathrm{kGs}$ wiggler [4]. Another experimental facility of VEPP-4M uses bremsstrahlung $\gamma$-rays from the movable beam scraper inserted in the halo of the circulating electron beam for test experiments with HEP detector components [8].

\section{VEPP-2000}

The VEPP-2000 collider [9, 10] operates at the energy range of $160 \div 1000 \mathrm{MeV}$ with two detectors: SND and CMD-3. The experimental program covers R measurements, studies of hadronic final states, mesons and theirs excitations, and nucleon electromagnetic formfactor at threshold, etc. $[11,12]$. The main design collider parameters are listed in Table 3 . In Fig. 3 one can find the collider layout.

Table 3. VEPP-2000 main design parameters (at $E=1 \mathrm{GeV}$ ).

\begin{tabular}{|l|l|l|l|}
\hline Circumference, $C$ & $24.39 \mathrm{~m}$ & Beam energy range, $E$ & $150-1000 \mathrm{MeV}$ \\
\hline Number of bunches & $1 \times 1$ & Particles per bunch, $N$ & $1 \times 10^{11}$ \\
\hline Beta-functions at IP, $\beta_{x, y}^{*}$ & $8.5 \mathrm{~cm}$ & Betatron tunes, $v_{x, y}$ & $4.1,2.1$ \\
\hline Beam-beam parameters, $\xi_{x, z}$ & 0.1 & Luminosity, $L$ & $1 \times 10^{32} \mathrm{~cm}^{-2} \mathrm{~s}^{-1}$ \\
\hline
\end{tabular}
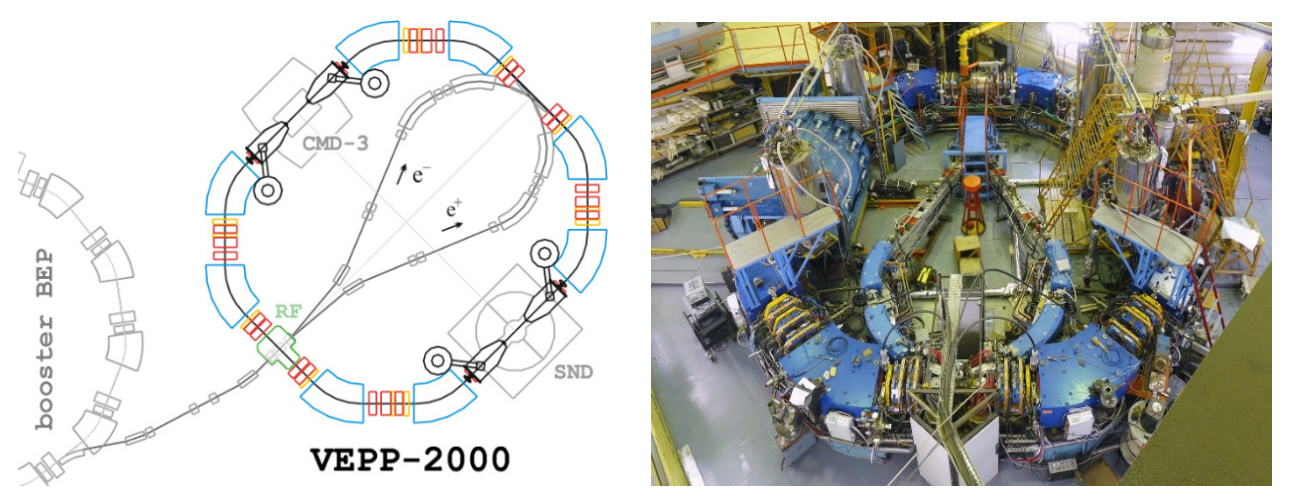

Fig. 3. VEPP-2000 schematic layout (left) and photo (right).

The VEPP-2000 is a small single-ring machine for collisions of $1 \times 1$ bunches. In order to increase luminosity VEPP-2000 exploits the round beam concept (RBC) [13]. This novel approach yields the significant beam-beam limit enhancement [14]. During first operation phase the scan in all energy range was done. Apart from partial integrability in beam-beam 
interaction the RBC gives a significant benefit in the Touschek lifetime when compared to traditional flat beams. This results in the ability of VEPP-2000 to operate at an energy as low as $160 \mathrm{MeV}$ - the lowest energy ever obtained in $\mathrm{e}^{+} \mathrm{e}^{-}$colliders.

The averaged over $10 \%$ of best runs luminosity logged by CMD-3 detector in 20102013 is shown in Fig. 4 (left) with dark-red points. The dashed line shows the beam-beam limited luminosity for a fixed machine lattice (energy scaling law $\mathrm{L} \propto \gamma^{4}$ ). It was successfully exceeded due to $\beta^{*}$ reduction to $4 \div 5 \mathrm{~cm}$ available at low energies. At middle energies after thorough machine tuning the beam-beam parameter achieved the maximal value of $\xi \sim 0.12$ per one IP during regular work breaking a world record [14].

The luminosity of VEPP-2000 at top energies was limited by insufficient $\mathrm{e}^{+}$production rate and necessity of acceleration at VEPP-2000 ring. In order to achieve the design luminosity the machine was stopped in 2013 for upgrade of the whole injection chain. The complex was linked up via a $250 \mathrm{~m}$ beamline K-500 to the IC. In addition, BEP was upgraded to provide top-up injection up to $1 \mathrm{GeV}$ [15].
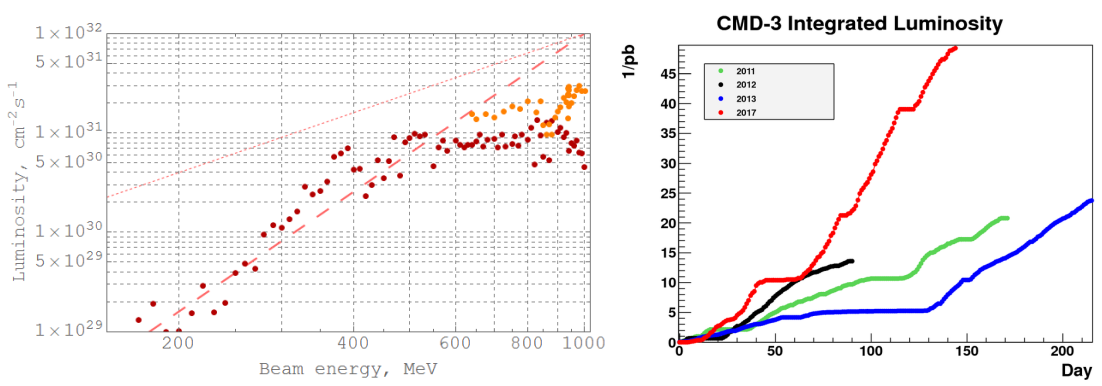

Fig. 4. Left: achieved averaged luminosity at CMD-3; orange dots show luminosity after complex upgrade. Right: Luminosity integral collected by CMD-3.

After beam arrival and complex recommissioning [16] the data collection was resumed in 2017. The achieved positron stacking rate at BEP increased up to $2 \times 10^{8} \mathrm{e}^{+} / \mathrm{sec}$ that exceeds in one order of magnitude former level. The first run was dedicated to high energy region. Fig. 4 presents the increased luminosity (see orange dots in left part) and the integrated by CMD-3 luminosity (red points in right part) in comparison with previous runs. Maximum peak luminosity achieved $4 \times 10^{31} \mathrm{~cm}^{-2} \mathrm{~s}^{-1}$ that is still more than twice lower than design value. Further increase of the beam currents and therefore the luminosity is currently prevented by the flip-flop effect that significantly reduces the beam lifetime [14].

The collected data distribution vs energy is shown in Fig. 5. Apart from regular scan significant data was collected at the nucleon threshold and slightly above design top energy at $1003.5 \mathrm{MeV}$ per beam relevant to $\mathrm{D}^{* 0}$ meson mass.
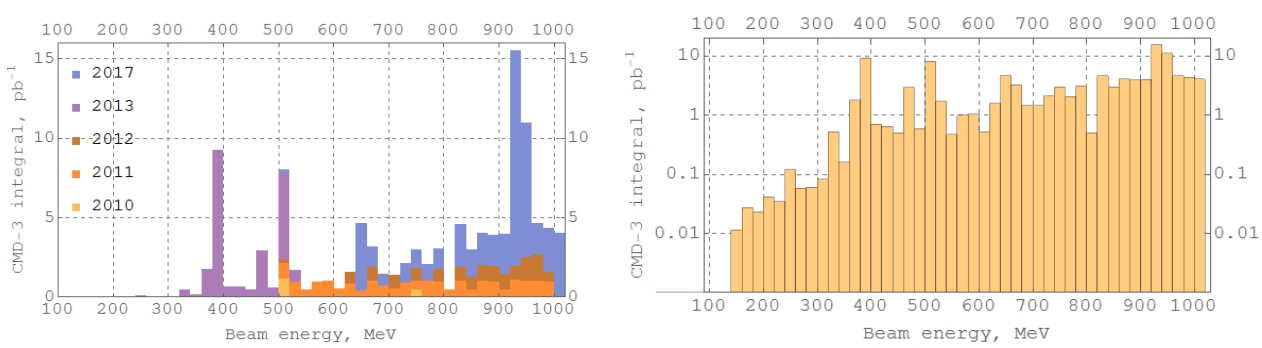

Fig. 5. Total luminosity integral collected by CMD-3 energy distribution. 


\section{Super Charm Tau Factory Project}

The main BINP future project for HEP is the Super Charm Tau factory [17]. It is an $\mathrm{e}^{+} \mathrm{e}^{-}$ collider with the beam energy range from 1 to $2.5 \mathrm{GeV}$ with extremely high luminosity $\left(\sim 10^{35} \mathrm{~cm}^{-2} \cdot \mathrm{s}^{-1}\right)$ and longitudinal polarization of electron beam at the IP. The facility can study the tau leptons, charmed particles and light quark spectroscopy in a unique manner.

The factory (Fig. 6) will consist of the $1.5 \mathrm{GeV}$ electron injector with the polarized and unpolarized photo-guns, the $1.5 \mathrm{GeV}$ positron injector with the damping ring, common for both beams $1 \mathrm{GeV}$ linac and the double ring collider. The main parameters are listed in Table 4.

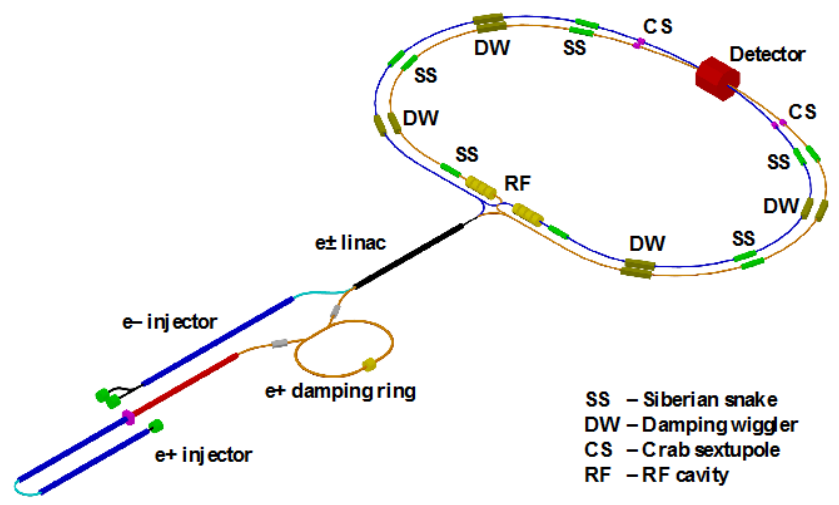

Fig. 6. Super Charm Factory layout.

Table 4. The main parameters of the Super Charm Tau Factory.

\begin{tabular}{|c|c|c|c|c|c|}
\hline Energy & 1.0 & 1.5 & 2.0 & 2.5 & GeV \\
\hline Circumference & \multicolumn{4}{|c|}{813.112} & $\mathrm{~m}$ \\
\hline Betatron tunes & \multicolumn{4}{|c|}{$43.54 / 40.57$} & \\
\hline Synchrotron tune & $6.71 \cdot 10^{-3}$ & $1.04 \cdot 10^{-2}$ & $1.06 \cdot 10^{-2}$ & $8.21 \cdot 10^{-3}$ & \\
\hline Damping wiggler field & 61.6 & 46.5 & 32.6 & 9.53 & kGs \\
\hline RF voltage & 0.305 & 0.895 & 1.239 & 0.993 & MV \\
\hline Damping times & \multicolumn{4}{|c|}{$30 / 30 / 15$} & $\mathrm{~ms}$ \\
\hline Coupling & \multicolumn{4}{|c|}{$0.5 \%$} & \\
\hline Emittances & \multicolumn{4}{|c|}{$8 / 0.04$} & $\mathrm{~nm} \cdot \mathrm{rad}$ \\
\hline Energy spread & $1.1 \cdot 10^{-3}$ & $1.1 \cdot 10^{-3}$ & $9.3 \cdot 10^{-4}$ & $7.2 \cdot 10^{-4}$ & \\
\hline Bunch length & 1.8 & 1.2 & 1.0 & 1.0 & $\mathrm{~cm}$ \\
\hline Particles per bunch & \multicolumn{4}{|c|}{$7.1 \cdot 10^{10}$} & \\
\hline Number of bunches & \multicolumn{4}{|c|}{406} & \\
\hline Beta functions at IP & \multicolumn{4}{|c|}{$4 / 0.08$} & $\mathrm{~cm}$ \\
\hline Crossing angle & \multicolumn{4}{|c|}{60} & $\operatorname{mrad}$ \\
\hline Piwinsky angle & 30.3 & 20.2 & 16.8 & 16.8 & \\
\hline Beam-beam parameters & $0.002 / 0.135$ & $0.003 / 0.135$ & $0.003 / 0.121$ & $0.003 / 0.097$ & \\
\hline Luminosity & $0.56 \cdot 10^{35}$ & $0.86 \cdot 10^{35}$ & $1.02 \cdot 10^{35}$ & $1.02 \cdot 10^{35}$ & $\mathrm{~cm}^{-2} \mathrm{~s}^{-1}$ \\
\hline
\end{tabular}

To achieve the design luminosity the Crab Waist scheme with a submillimeter vertical betatron function at the IP must be implemented. It is planned to install 4 superconducting 
wigglers $(1.6 \mathrm{~m}, 6.2 \mathrm{~T}, 35 \mathrm{~cm}$ period) in each ring to make the beam emittance and the damping time invariant in the full energy range. It allows to establish linear dependence of the luminosity on the beam energy. To control electron beam polarization 5 Siberian snakes with $2 \mathrm{SC}$ solenoids (1.9 m length, $7 \mathrm{~T}$ field) will be installed in the electron ring.

The superconducting final focus doublet with anti-solenoids will be integrated into universal magnetic detector with $1 \mathrm{~T}$ longitudinal field.

\section{Acknowledgements}

This work was supported by Russian Science Foundation under project N 14-50-00080.

\section{References}

1. D. Berkaev et al., VEPP-5 Injection Complex: two colliders operation experience, Proc. IPAC'17, Copenhagen, Denmark (2017), pp. 2982-2984.

2. F. Emanov et al., Feeding BINP Colliders with the new VEPP-5 Injection Complex, Proc. RuPAC'16, St.Peterburg, Russia (2016), pp. 56-60.

3. E.B. Levichev, Status and upgrade of the VEPP-4 storage-ring facility, Phys. Part. Nucl. Lett. 137 (2016), pp. 876-883.

4. P.Piminov et al., Synchrotron radiation research and application at VEPP-4, Physics Procedia 84 (2016), pp. 19-26.

5. V.V. Neufeld, V.V. Petrov, Positron injector of the VEPP-4M collider, Phys. Part. Nucl. Lett. 137 (2016), pp. 893-896.

6. P.Piminov, Status of the electron-positron collider VEPP-4, Proc. IPAC'17, Copenhagen, Denmark (2017), pp. 2985-2988.

7. V.V. Anashin et al, The KEDR detector, Phys. Part. Nucl. 444 (2013), pp. 657-702.

8. V.S. Bobrovnikov et al., Extracted electron and gamma beams in BINP, JINST 9 (2014) C08022.

9. D. Shwartz et al., Implementation of Round Colliding Beams Concept at VEPP-2000, Proc. eeFACT'16, Daresbury, UK (2016), pp. 32-38.

10. P. Shatunov et al., High Luminosity at VEPP-2000 Collider With New Injector, Proc. IPAC'17, Copenhagen, Denmark (2016), pp. 2989-2991.

11. G.V. Fedotovich et al., Overview of the CMD-3 recent results at $e+e-$ collider VEPP2000, EPJ Web Conf. 130 (2016), 01014.

12. I.B. Logashenko, Measurement of hadronic cross-sections at VEPP-2000, PoS ICHEP2016, Chicago, USA (2016), p.544.

13. V.V. Danilov et al., The Concept of Round Colliding Beams, Proc. EPAC'96, Sitges, Spain (1996), pp. 1149-1151.

14. D. Shwartz et al., Recent Beam-beam Effects and Luminosity at VEPP-2000, Proc. IPAC'14, Dresden, Germany (2014), pp. 924-927.

15. D. Shwartz et al., Booster of Electrons and Positrons (BEP) Upgrade to $1 \mathrm{GeV}$, Proc. IPAC'14, Dresden, Germany (2014), pp. 102-104.

16. D. Shwartz et al., Recomissioning and Perspectives of VEPP-2000 e+e-Collider, PoS ICHEP2016 (2016), p.054.

17. A.E.Bondar et al., Project of a Super Charm-Tau factory at the BINP in Novosibirsk, Phys. Atom. Nucl. 76 (2013) pp. 1072-1085. 